\title{
Philosophiques
}

\section{Banalité du mal et sens du devoir chez les administrateurs de l'extermination}

\section{Jean-Ernest Joos}

Volume 19, numéro 1, printemps 1992

URI : https://id.erudit.org/iderudit/027172ar

DOI : https://doi.org/10.7202/027172ar

Aller au sommaire du numéro

Éditeur(s)

Société de philosophie du Québec

ISSN

0316-2923 (imprimé)

1492-1391 (numérique)

Découvrir la revue

Citer cet article

Joos, J.-E. (1992). Banalité du mal et sens du devoir chez les administrateurs de l'extermination. Philosophiques, 19(1), 61-74. https://doi.org/10.7202/027172ar
Résumé de l'article

Dans son rapport sur le procès d'Eichmann, Hannah Arendt propose le concept de banalité du mal pour caractériser le comportement des fonctionnaires allemands qui ont rendu possible l'Extermination des juifs. La banalité du mal désigne la perte du sens de la responsabilité politique au profit d'un simple " sens du devoir » à l'égard de l'Etat quel qu'il soit. Pourtant, selon l'historien Raoul Hilberg, ce qui frappe dans le processus de l'Extermination c'est la remarquable autonomie des services administratifs impliqués par rapport à la sphère politique. Comprendre comment cette autonomie peut se concilier avec un non-engagement politique, telle est la question philosophique ici posée. 


\title{
BANALITÉ DU MAL ET SENS DU DEVOIR CHEZ LES ADMINISTRATEURS DE L'EXTERMINATION
}

\author{
par Jean-Ernest Joos
}

\begin{abstract}
Résumé. Dans son rapport sur le procès d'Eichmann, Hannah Arendt propose le concept de banalité du mal pour caractériser le comportement des fonctionnaires allemands qui ont rendu possible l'Extermination des juifs. La banalité du mal désigne la perte du sens de la responsabilité politique au profit d'un simple " sens du devoir " à l'égard de l'État quel qu'il soit. Pourtant, selon l'historien Raoul Hilberg, ce qui frappe dans le processus de l'Extermination c'est la remarquable autonomie des services administratifs impliqués par rapport à la sphère politique. Comprendre comment cette autonomie peut se concilier avec un non-engagement politique, telle est la question philosophique ici posée.
\end{abstract}

ABSTRACT. The concept of banality of evil, as proposed by Hannah Arendt in her report on Eichmann's trial, serves the purpose of understanding the role and attitude of german bureaucrats regarding the Extermination process. This concept relates to a sense of duty lacking of personal judgement and political responsability. However, the description Raoul Hilberg (an historian) gives of the Bureaucracy of extermination reveals remarkably self-governing actions. How does this self-government and the absence of political intentions can coexist in a same person, in a same institution?

La mise en place du processus de l'Extermination des Juifs par l'Allemagne nazie a non seulement trahi l'essence d'une bureaucratie dont la finalité est celle d'une rationalité instrumentale, mais a assigné à l'individu un statut tout à fait particulier au sein des institutions. On a vu des individus participer à une activité sans que leurs motivations ou leurs 
personnalités ne soient à la mesure de l'horreur du résultat produit. Quelle a bien pu être la nature des liens socio-politiques de l'individu aux organismes auxquels il appartenait pour qu'une telle dissociation entre les motivations personnelles et l'effet final ait été possible? Pour caractériser la nature de ces liens, Hannah Arendt propose une radicalisation de la notion d'un sens du devoir sous le concept de «banalité du mal». C'est la pertinence de ce concept qu'il s'agit d'analyser, en reposant la question très limitée et très précise de l'incidence des conditions subjectives du processus de l'Extermination sur l'organisation objective du processus. Il semblerait bien que ce soit là que réside une des grandes nouveautés de l'administration de l'Extermination: elle aurait modifié le mode selon lequel l'individu se définit subjectivement au sein de l'État, face à sa participation aux activités et réalisations de l'État.

\section{La bureaucratie dans le processus de l'Extermination}

Lorsqu'on s'interroge sur les mécanismes de destruction ayant permis l'Extermination des Juifs par l'Allemagne nazie, on s'aperçoit qu'ils font intervenir tous les niveaux de gouvernement et toutes les sphères de l'État. On ne saurait limiter la machine de destruction aux organismes explicitement reliés au mouvement nazi (Gestapo, et S.S...), car elle fait intervenir aussi bien pour les coûts de l'opération, le ministère des Finances que l'administration des chemins de fer sans laquelle les Juifs n'auraient jamais pu être déplacés, ou encore la police de l'ordre, corps traditionnel, dont l'existence était bien antérieure à celle du parti nazi, et qui a servi à de nombreuses fonctions: identifier les Juifs, les répertorier, les arrêter. Bref, comme le déclare l'historien Raoul Hilberg, " parler de la machine de destruction, c'est parler de l'État allemand considéré dans une de ses fonctions spécialisées $»^{1}$. Or il y a là de quoi s'étonner. Que des fonctionnaires chargés d'une tâche précise nécessaire au fonctionnement de tout État moderne, se retrouvent comme des rouages de l'Extermination, sans pourtant quitter leurs fonctions, voilà une des énigmes que pose l'Allemagne nazie.

On peut à la rigueur comprendre par une adhésion idéologique les motivations de tel ou tel individu recruté pour assurer explicitement un rôle dans le processus de destruction, mais on voit plus difficilement celles d'un comptable, d'un chef de gare, d'un gendarme qui ont toujours fait leur métier et qui continueront de le faire consciencieusement même si leurs 
actes "professionnels" ont pris avec la mise en place de l'Extermination un sens radicalement nouveau. Le comportement de ces derniers est d'autant plus énigmatique que les justifications qu'ils donnèrent après coup de leurs actes passés sont souvent hautement contradictoires: nous ne savions pas et, de toute façon, nous n'avons jamais fait que notre devoir. Ils renvoient ainsi le sens et la responsabilité de leurs actes à des dirigeants dont les intentions peuvent être attribuées, de façon claire et rassurante, à une haine raciale, démente et inhumaine, dont ils peuvent aisément se dissocier. Et pourtant sans eux, jamais $l^{\prime}$ Extermination n'aurait été matériellement possible. Comme le révèle en détail Hilberg ${ }^{2}$, ils n'ont pas seulement collaboré au processus de destruction, ils l'ont administré, systématisé, en ont délimité rationnellement les conditions de possibilité àl'intérieur du fonctionnement "normal " d'une société. Sans eux, l'Extermination serait restée un projet délirant. C'est donc à eux qu'il faut s'intéresser, à ces administrateurs, qui n'ont le plus souvent été, ni les initiateurs du projet, ni ceux qui à l'autre extrémité de la chaîne ont opéré les camps d'Extermination mais qui, entre ces deux activités dont la criminalité étaient évidentes et qui se fusionnaient souvent, ont mis au service des uns et des autres tout le potentiel de la société.

\section{Le sens du devoir conçu comme perte de l'autonomie du jugement}

Pour comprendre les agissements de ces bureaucrates de l'Allemagne nazie, Hannah Arendt a forgé le concept de la "banalité du mal" à l'occasion de son rapport controversé sur le procès d'Eichmann à Jérusalem. Il ne sera pas question ici de savoir si le portrait qu'elle fait de l'homme est juste ou non, mais si ce qu'elle dit à son propos peut s'appliquer à ces fonctionnaires que l'on examine ici. Â son procès, Eichmann déclara, à la surprise de ses juges, qu'il avait toujours vécu sur les principes moraux de Kant, sauf lorsqu'il avait été chargé de l'application de la "Solution finale». Et il fournit effectivement une formulation à peu près juste de l'impératif catégorique. Cette intervention étonnante d'Eichmann sert à Arendt de point de départ à une interrogation qui porte sur ce qu'il y a de kantien dans l'attitude d'Eichmann ${ }^{3}$. C'est le sens "moral" du devoir. Eichmann aurait agi par devoir, par respect pour la loi. Pour lui, obéir à la loi aurait signifié " agir comme si on était le législateur de la loi à laquelle on obéit "4. C'est ce qui explique alors que la "Solution finale" ait été appliquée par tous les fonctionnaires allemands avec 
tant de zèle et d'efficacité. Leur comportement ne s'explique donc pas par un antisémitisme excessif, mais plutôt par un respect démesuré de l'ordre qui les a conduit à identifier leur propre volonté au principe et à la source de la loi. Bien entendu, cette source n'est pas la raison pratique comme dans la pensée de Kant, mais l'État. Arendt juge que les agissements d'Eichmann répondaient plutôt à un impératif qu'elle reconstruit comme suit: "Agissez comme si le principe de vos actes étaient le même que celui des législateurs ou des lois du pays ". Et cette formule recoupe un impératif qui a été inventé par un des idéologues du Troisième Reich, Hans Frank, et qui s'énonçait ainsi: "Agissez de telle manière que le Führer, s'il avait connaissance de vos actes, les approuverait $n^{5}$.

À l'appui de sa thèse, Arendt relate un épisode du procès où Eichmann avoua avoir rendu service à un cousin demi-juif et à un couple juif. Ces deux exceptions à la loi ne constituaient pas pour Eichmann des circonstances atténuantes, mais au contraire aggravantes, car elles le privaient de ce qui représentait à ses yeux sa seule justification et le seul sens possible de ses actes, à savoir le sens du devoir. Et de fait, on sait que de nombreux fonctionnaires ont dérogé à la loi pour venir en aide à des Juifs proches, à tel point qu'Himmler dût rappeler à l'ordre les Allemands en leur faisant comprendre qu'il n'était pas acceptable que "nos 80 millions de dignes Allemands" aient chacun leur "Juif honnête " ${ }^{6}$. Et Arendt comme Hilberg de remarquer que ces manifestations d'humanité n'ont pas entravé le processus d'Extermination, mais bien plutôt l'ont rendu possible ${ }^{7}$. En effet, elles permettaient aux fonctionnaires de faire taire la voix de leur conscience, en les convainquant que leurs actes ne répondaient à aucune motivation personnelle, qu'ils n'auraient pas agi ainsi si cela avait dépendu d'eux, et donc qu'ils ne faisaient qu'obéir en bons sujets de l'État aux ordres donnés. Ils ont pu ainsi redoubler de zèle tout en renvoyant la responsabilité de leurs actes sur d'autres. C'est cela qu'Arendt appelle la "banalité du mal", l'absence de toute intention de faire le mal, l'absence effrayante de toute intention propre à un sujet autonome, la disparition du sens moral de la responsabilité individuelle.

Cette perte de l'autonomie qui permet à des hommes de commettre avec indifférence les gestes les plus inhumains est attribuable tout d'abord à l'essence de la bureaucratie qui réduit les hommes à des rouages administratifs anonymes, et 
qui instaure le «règne de Personne " $^{8}$. Cette déshumanisation par la bureaucratie trouve, pour Arendt, son expression la plus achevée dans la forme politique du totalitarisme. Pour le régime totalitaire tel que l'analyse Arendt, «le libre consentement constitue un obstacle aussi grand que la libre opposition ${ }^{9}$. Il ne faut pas laisser aux hommes la liberté et le désir de croire en l'idéologie, car il resterait alors un écart dangereux pour la domination totalitaire entre les normes de la conduite individuelle et le règne de la loi totalitaire ${ }^{10}$. Il ne suffit pas d'éduquer les hommes à juger dans le sens de l'idéologie, il s'agit plus fondamentalement de les priver de la faculté même de juger ${ }^{11}$. De l'idéologie, seule compte la logique interne, nullement son origine, ou sa fin, ou ses rapports à la réalité, ou les hommes à qui elle semble s'appliquer. On ne doit pas pouvoir juger de sa valeur ou de son efficacité, de sa réussite ou de son échec face à des objectifs quelconques, de son adéquation ou de son inadéquation à la réalité naturelle et sociale. Alors, l'idéologie totalitaire peut s'imposer inconditionnellement à l'homme. Arendt parle "d'auto-contrainte de la logique totalitaire" qui "détruit chez l'homme la faculté $\mathrm{d}^{\prime}$ expérimenter et de penser aussi certainement que celle d'agir $»^{12}$. Elle peut donner à partir de là le portrait-type du sujet idéal du règne totalitaire: il n'est

ni le nazi convaincu, ni le communiste convaincu, mais l'homme pour qui la distinction entre fait et fiction (i.e. la réalité de $l^{\prime}$ expérience) et la distinction entre vrai et faux (i.e. les normes de la pensée) n'existent plus ${ }^{13}$.

On retrouve ainsi dans cette analyse du totalitarisme les éléments qui serviront quelques années plus tard à Arendt pour son rapport sur Eichmann.

Le concept de banalité du mal revêt alors une valeur politique très particulière. Il ne désigne pas une certaine politique, mais bien la perte du sens même du politique. La perte du sens du politique désigne tout d'abord le retrait de l'individu de la vie politique, l'individu préférant ses intérêts personnels à une implication dans la communauté. Ce mouvement de retrait, Arendt le fait remonter aussi loin qu'aux fondements de la pensée libérale. Plus profondément, la perte du sens du politique désigne, avec le totalitarisme, l'anéantissement par la politique elle-même de ce qui constitue son fondement propre: la sécurité et la survie de l'humanité ${ }^{14}$. La politique cesse d'être une condition de la vie en commun, au contraire 
elle neutralise désormais la possibilité d'un "agir ensemble". Or cet "agir ensemble» suppose que « les décisions concernant le juste et le faux dépendent du choix de la compagnie avec laquelle nous entendons vivre ${ }^{15}$. La banalité du mal désigne alors, selon les mots d'Arendt, "l'incapacité ou le refus de se rapporter aux autres par le biais du jugement ${ }^{16}$. Et $c^{\prime}$ est bien cette incapacité ou ce refus qui se manifeste lorsque le lien social se trouve réduit à un lien de devoir dans un système bureaucratique dont la cohésion se base sur la finalité technologique. La banalité du mal révélée par le processus de l'Extermination nous oblige à nous interroger sur les racines mêmes du politique qui réside dans «notre capacité à partager le monde avec autrui $»^{17}$.

Paradoxalement, cette notion de dépolitisation qui recouvre tout à la fois la perte de l'individualité et la perte du sens de la communauté permet à Arendt de réintroduire la responsabilité dans son jugement sur les bureaucrates nazis. Eichmann reste responsable et coupable, malgré son absence d'intention de faire le mal. Il l'est parce qu'il a détruit objectivement les conditions de la communauté humaine, il a refusé de partager le monde avec d'autres hommes ${ }^{18}$. Il est donc responsable devant l'humanité d'avoir perdu le sens de l'humain, d'être devenu cette chose anonyme, n'obéissant que par sens du devoir et sens de la logique aux lois les plus inhumaines ${ }^{19}$.

\section{La double attitude de soumission et d'autonomie du fonctionnaire}

Pourtant, ce portrait du fonctionnaire de l'Allemagne nazie fourni par Arendt soulève quelques interrogations à la lumière de ce que l'histoire nous apprend du rôle de la bureaucratie dans le processus $d$ 'Extermination. On appuiera nos réflexions sur les analyses de l'historien Raoul Hilberg. Deux faits ici sont troublants. Tout d'abord, s'il est bien certain que la notion de devoir a joué un rôle essentiel, il est cependant aussi important de constater que le processus n'aurait pu se réaliser "si tout le monde avait attendu les ordres pour agir ${ }^{20}$. Arendt néglige ici la nature tout à fait particulière de la transmission des ordres sous l'Allemagne nazie, notamment au moment de la mise en acte de l'Extermination. L'image du fonctionnaire zélé qui obéit à une loi clairement énoncée est douteuse. Le deuxième fait troublant relevé par Hilberg est que ces bureaucrates et technocrates de $1^{\prime}$ Extermination ont été des "inventeurs " ${ }^{21}$. Ils n'ont pas seulement mis une bureaucratie et une technologie au service du régime, ils les ont surtout détournées de leurs 
finalités initiales, ils leur ont inventé des usages inédits et des potentialités imprévisibles. Et cela n'est pas seulement vrai pour les instruments de meurtre collectif (dont la transformation des camions Saurer en chambres à gaz donne un bon exemple ${ }^{22}$ ) mais aussi pour tous les cheminots qui ont dû faire face aux considérables difficultés de ces déplacements de population en direction des camps. Tous, ils ont eu à passer par les différentes démarches de l'invention: imaginer, expérimenter, modifier... Tout en suivant Arendt dans son intuition sur la perte du sens politique, on parlera de perversion plutôt que de banalité du mal et la perversion désignera ici la capacité du sujet fonctionnaire à être double, à donner un sens à son acte face à lui-même et face aux autres tout en accomplissant une fin objectivement toute autre. Et il s'agira de montrer de plus que cette perversion n'est pas le propre du fonctionnaire, mais est un élément du fonctionnement de l'administration lors de l'Extermination.

La première anomalie à considérer est le statut de l'ordre légal d'Extermination. On sait que les historiens débattent longuement de l'origine du processus de l'Extermination. Les uns adoptant une thèse intentionnaliste mettent l'accent sur le projet de Hitler et sur sa mise en application, en accord avec une volonté explicite du régime nazi. Les autres qui se définissent comme fonctionnalistes considèrent que la mise en place de l'Extermination s'est faite progressivement et de manière improvisée en réponse aux pressions idéologiques créées par la «promesse » d'une Europe «judenrein » et aux difficultés liées à la guerre sur le front de $1^{\prime}$ Est $^{23}$.

Ce débat entre historiens est symptomatique de la nature politique de l'ordre d'exterminer. Il est bien certain que Hitler n'a jamais caché, que ce soit dans ses déclarations publiques ou privées, son intention d'exterminer les Juifs. Mais il est presque tout à fait certain aussi que l'ordre d'exterminer les Juifs n’a jamais été signé par Hitler. II ne s'agissait très probablement que d'une directive orale, de la manifestation d'une intention. Et tout le processus a fonctionné sur la transmission essentiellement orale des ordres. Et Hilberg d'affirmer qu'ultimement « les ordres eux-mêmes n'étaient plus nécessaires ${ }^{24}$. De plus, ces ordres étaient souvent imprécis et ne tenaient pas compte de difficultés quasi insurmontables. On a vu alors des fonctionnaires prendre l'initiative, résoudre les problèmes, concrétiser les ordres par des propositions qu'ils soumettaient à leurs supérieurs ${ }^{25}$. 
Politiquement, les ordres en question ne peuvent être considérés comme des lois. Une loi contient toujours en elle-même sa propre réglementation, ses propres conditions d'application qui lui donnent une emprise sur la réalité. Rien de tel ici. L'ordre hitlérien ne serait resté qu'une loi délirante sans l'organisation et les initiatives de la bureaucratie allemande. Il aurait même été possible de $s^{\prime} y$ soustraire sans désobéir, en puisant dans la force passive de résistance dont toute bureaucratie est capable lorsqu'on lui demande de résoudre des problèmes difficiles qui dépassent sa compétence habituelle (comme ce fut le cas tout particulièrement en Italie, ou encore en Hongrie ou en Bulgarie ${ }^{26}$ ).

Le portrait que Raoul Hilberg propose du bureaucrate allemand fait donc référence à une certaine autonomie, limitée bien sûr par le contexte totalitaire, mais difficilement compatible avec le concept de banalité du mal. Cette autonomie n'invalide pas la thèse du devoir invoquée par Arendt, mais elle modifie le sens de ce devoir. Il y a dans la formule de Hans Frank citée par Arendt une dimension qu'elle ne relève pas. Dans la traduction que Hans Frank opère du sujet politique en sujet moral, il y a la notion d'une obéissance au Führer qui doit se faire même en l'absence d'une loi énoncée. Comme dans l'impératif moral de Kant, il revient à chacun de trouver la loi à laquelle il doit se soumettre. "Agissez de telle manière que le Führer, s'il avait connaissance de vos actes, les approuverait ». Et le sujet n'a que les intentions de Hitler et l'idéologie pour se guider. Cette formule peut s'interpréter selon le concept qu'Arendt donne du totalitarisme comme une assimilation de la volonté individuelle à la logique de l'idéologie. Mais de fait, l'idéologie ne pouvait déterminer les agissements individuels, pas plus que l'État, incarné par Hitler, ne contrôlait les différents services administratifs qui ont toujours conservé une très grande autonomie, même dans le processus d'Extermination ${ }^{27}$. La formule de Hans Frank peut alors s'entendre autrement: pour un sujet nazi qui a le sens du devoir toute initiative personnelle prend aussitôt la valeur d'un devoir envers le Führer. C'est ainsi que les administrateurs de l'Extermination ont pu répondre aux ordres émis par un effort personnel de rationalisation tout en considérant que leurs initiatives ne constituaient que l'expression du devoir à accomplir. Et de fait, l'Extermination n'aurait été possible sans la notion de devoir qui faisait taire la conscience morale, ni sans l'intervention active de la volonté et de l'intelligence de chacun qui rendait le système efficace. 


\section{Le double sens de l'activité dans l'administration de l'Extermination}

Notre hypothèse suppose une perversion du fonctionnaire par rapport à sa fonction dans l'administration. D'une part, le sens du devoir lui permettait de rejeter la responsabilité de ses actes sur ses supérieurs qui donnaient les ordres, mais d'autre part l'autonomie qu'il conservait lui permettait de se dissocier de l'ensemble du processus administratif et tout particulièrement de la finalité de celui-ci, et ainsi de définir le sens de son acte uniquement à l'intérieur de la sphère administrative qui le concerne. Il peut ainsi garder la satisfaction du travail bien fait, même après que le but dernier de son travail eût été révélé à la face du monde comme étant l'Extermination, car il peut toujours soutenir qu'il n'y avait d'autre sens à ses actes que celui qu'il leur avait donné.

Cette attitude est fort bien révélée par Claude Lanzmann lorsque dans son film Shoah il confronte d'anciens fonctionnaires du nazisme aux conséquences de leurs agissements et les interroge sur le sens qu'ils donnaient à l'époque à leurs actes. Ainsi, Franz Grassler, adjoint au commissaire nazi du ghetto de Varsovie, peut se permettre d'affirmer encore aujourd'hui que sa tâche consistait uniquement à "maintenir en vie le ghetto ", même si la mort des Juifs faisait déjà partie d'une politique $d^{\prime}$ Extermination ${ }^{28}$. Bien sûr, il ignorait tout, dit-il, d'une politique d'ensemble à l'égard des Juifs. Hypocrisie? La question n'est plus là. La feinte ignorance de Grassler lui permet de se poser en simple témoin d'une histoire dont il a été l'acteur. Ainsi, Grassler pourra prendre des notes, avec une certaine fierté et un semblant d'intérêt pour l'histoire, sur sa propre participation au meurtre collectif que constituait le ghetto ${ }^{29}$, car cette mise en scène d'un Edipe qui découvre le sens de ce qu'il a fait ne s'accompagnera d'aucune révélation sur son rôle d'acteur. Pour le fonctionnaire nazi lié à ses actes par les liens du devoir, le savoir est dissocié de l'action. Le passage de l'ignorance au savoir ne le concerne alors qu'en tant que témoin. Cette dissociation entre le savoir et l'action reproduit en fait celle qui a rendu possible le processus d'Extermination. Au sein du processus, le fonctionnaire a toujours conservé la liberté de se rapporter subjectivement à ses actes en détournant leur sens de celui qu'ils avaient par rapport à leurs effets réels.

Ce détournement du sens de son acte est rendu d'autant plus aisé au fonctionnaire que le processus d'Extermination 
constitue par lui-même un détournement de la finalité normale de l'administration. Il suffit alors au fonctionnaire de revendiquer sa fonction "normale" pour se dissocier de la finalité que l'Extermination a donné à l'administration à laquelle il appartient. Et ce passage d'un mode à l'autre est rendu plus aisé par son statut "d'inventeur». On en a un bon exemple dans la déportation des Juifs par l'administration des chemins de fer du Reich. Elle s'est faite en détournant la catégorie administrative de "train spécial " qui servait normalement à gérer les touristes, ou les voyages officiels et à l'utiliser pour transporter les Juifs sans nuire au réseau ${ }^{30}$. Cela constituait déjà en soi une idée originale que seul un agent des chemins de fer aurait pu mettre au point. Le grand avantage du système est qu'il permettait de plus à la Reichsbahn de se faire payer ces «transports exceptionnels » de façon à assurer la stabilité du réseau $^{31}$. Or aucun cheminot ne pouvait ignorer qu'un «train spécial " signifiait train vers la mort.

Et pourtant, le signifiant "train spécial" a toujours été un signifiant à double sens: un sens à l'intérieur de l'univers des chemins de fer, un autre dans le processus global de l'Extermination. Rien dans l'ordre de route de ces "trains spéciaux", pas même la mention "secret", ne nommait ce que ces trains accomplissaient. L'absence de classement particulier ou de précaution de dissimulation protégeaient le double sens de la désignation ${ }^{32}$. Il n'est pas important que le fonctionnaire ait su ou non la destination et la raison d'être de ses trains. Par contre, il est essentiel que ce savoir ne puisse jamais recevoir de reconnaissance objective ou de dénonciation, que ce soit aujourd'hui ou à l'époque. Il en est de même pour toutes les appellations codées qui ne servaient à garder aucun secret puisqu'il était nécessaire que toute l'administration les comprenne pour que le système fonctionne efficacement. Le rôle de ces faux secrets était plutôt de permettre à chacun de faire sa propre interprétation du mot codé, de lui donner un sens limité à sa sphère administrative et dissocié de la fin dernière de l'ensemble du processus administratif d'Extermination. Ainsi, un nazi, à son procès a pu affirmer que "l'expression

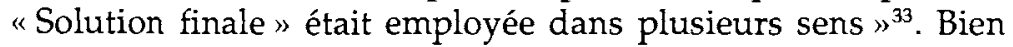
entendu, cette expression, comme toutes les autres formules codées, ne pouvait avoir dans le processus global qu'une seule signification décisive: l'Extermination. Mais la perversion du système est que le sens communiqué ne fait jamais l'objet d'une reconnaissance commune, et donc que les individus 
sont connectés par des liens sociaux dans lesquels ils ne sont pas obligés de se reconnaître. Ainsi, dans l'administration nazie, chacun a parfaitement rempli son rôle dans le processus d'Extermination, sans avoir pour autant à identifier le sens de ses actes par rapport au sens de ce processus qu'il ne pouvait pourtant ignorer.

\section{La perversion du politique}

Il devient difficile de parler de banalité du mal. Certes, le concept du devoir est ici d'une certaine pertinence, mais uniquement dans la mesure où le sens du devoir est ce qui a permis à l'administrateur de l'Extermination de dissocier les conditions subjectives de son activité (notamment le savoir et l'intention) de la reconnaissance objective de ces mêmes conditions au sein du processus global. En concluant trop vite à l'absence d'intention chez le fonctionnaire nazi, Arendt reproduit cette dissociation dans laquelle il faut voir la tournure perverse que prend la soumission au devoir dans l'administration de la "Solution finale". Objectivement, le fonctionnaire ne fait que son devoir, mais subjectivement, il fait toujours plus que son devoir: il invente l'Histoire. La force du système est précisément de rendre impossible toute reconnaissance objective de la subjectivité des acteurs. De là à conclure que cette subjectivité ne rentre pas en ligne de compte, il n'y a qu'un pas qu'il ne faut pas franchir, sous peine de se laisser piéger par la perversion du système. Ainsi, il serait naïf de croire que les fonctionnaires nazis ne "pensent» pas, qu'ils ont perdu tout désir de se justifier. Au contraire, ils se justifient grâce aux justifications que le système leur a fourni a priori. Ce faisant, ils se déchargent d'avoir à rendre compte après coup des conséquences de leurs actes. Il n'est pas important de savoir s'ils croient ou non à ces justifications souvent simplistes. Leur force est de croire que personne ne peut savoir s'ils y croient ou non et donc que leurs justifications ont une valeur objective. Il ne faut pas non plus conclure que le fonctionnaire a perdu le sens de la contradiction, il résout plutôt la contradiction entre son engagement actif et sa passivité revendiquée, en faisant du premier une simple manifestation du second.

Il faut reconnaître cependant qu'Arendt avec ses notions de banalité du mal et de perte du sens du politique met le doigt sur la nouveauté du fonctionnement de l'administration nazie. Ce qui a changé avec cette administration, c'est le mode selon lequel l'individu se rapporte au tout politique auquel il 
appartient. Cela Arendt l'a bien compris. Mais là où elle voit une perte totale de toute pensée et engagement politiques chez l'individu, il faut plutôt reconnaître un système administratif qui pervertit le lien politique liant l'individu au tout et permet au contraire de mettre à profit, dans une action globale, les motivations personnelles de chacun, quelles qu'elles soient (quête de la reconnaissance historique ou sociale, opinions politiques diverses, haines quotidiennes, intérêts matériels, satisfaction à réussir l'impossible, etc.) $)^{34}$. Et toutes ces motivations, en elles-mêmes d'incidence limitée et parfois même divergentes, ont été, sous le couvert du devoir, mises au service d'un processus unique dans lequel personne n'a eu à reconnaître l'effet de sa volonté. Le devoir restait en effet le seul lien objectivement déterminable au résultat final, $l^{\prime}$ Extermination, dont la responsabilité pouvait alors être renvoyée soit à l'Histoire elle-même, soit à l'action de l'État, ou à la volonté de Hitler. $C^{\prime}$ est là la perversion du politique.

Philosophie

Collège Marie de France, Montréal

\section{NOTES}

1. Raoul Hilberg, La destruction des Juifs d'Europe, trad. Marie- France de Paloméra et André Charpentier, Paris, Fayard, 1985, p. 53.

2. R. Hilberg, op. cit. et "La bureaucratie de la solution finale », in L'Allemagne nazie et le génocide juif, Paris, Gallimard, Le Seuil, 1985.

3. Hannah Arendt, Eichmann à Jérusalem. Rapport sur la banalité du mal, édition revue et augmentée, trad. Anne Guérin, Gallimard, 1966, p. 152 et suivantes.

4. Ibid. p. 154.

5. Cité par Arendt, op. cit., p. 153.

6. R. Hilberg, La destruction des Juifs d'Europe, p. 886.

7. Ibid.

8. H. Arendt, Eichmann à Jérusalem, p. 316.

9. H. Arendt, Le système totalitaire, trad. par Jean-Lory Bourget, Robert Davreu et Patrick Lévy, Paris, Éditions du Seuil, 1972, p. 190.

10. Ibid. p. 206.

11. Ibid. p. 215. 
11. Ibid. p. 215.

12. Ibid. p. 225.

13. Ibid. p. 224.

14. Myriam Revault-d'Allonnes, "Lectures de la modernité: Heidegger, Carl Schmitt, Hannah Arendt ", Les Temps Modernes, février 1990.

15. Arendt cité par Etienne Tassin, "Sens et communauté: la lecture arendtienne de Kant ", in Les cahiers de philosophie, 4, Hannah Arendt. Confrontations, p. 95.

16. Ibid.

17. M. Revault D'Allones, op. cit., p. 106.

18. Arendt, Eichmann à Jérusalem, p. 305. Voir aussi "Responsabilité personnelle et régime dictatorial ", in Penser l'événement, Paris, Bélin, 1989.

19. Arendt précise dans la Vie de l'esprit en quoi consiste la perte du sens de l'humain. L'homme mauvais est celui qui n'entretient pas de dialogue silencieux avec lui-même, selon la définition qu'Arendt donne de la pensée, et qui, par conséquent, ne craint jamais de se contredire et ne possède jamais ni la possibilité ni le désir de se justifier. Or " une vie dépourvue de pensée... n'est pas tout à fait vivante ", dit Arendt. Aussi l'homme qui ne pense pas n'est-il pas tout à fait humain. H. Arendt, La Vie de l'esprit, trad. Lucienne Lotringer, Paris, P.U.F., 1981, vol. 1, pp. 216 et 217.

20. Hilberg, "La bureaucratie de la solution finale ", p. 227.

21. Claude Lanzmann, Shoah, Paris, Fayard, 1985, p. 94 et suivantes (dans l'édition du "Livre de poche").

22. Ibid. p. 130 et suivantes.

23. Le débat entre historiens est bien résumé par Enzo Traverso, "Auschwitz, l'Histoire et les historiens », Les Temps Modernes, juin 1990.

24. Hilberg, «La bureaucratie de la solution finale», p. 228.

25. Ibid. et Hilberg, La destruction des Juifs d'Europe, p. 884.

26. Hilberg, La destruction des Juifs d'Europe, p. 865, p. 872.

27. Hilberg, "La bureaucratie de la solution finale ", p. 220.

28. Claude Lanzmann, Shoah, p. 228 et suivantes.

29. Ibid. p. 222.

30. Claude Lanzmann, Shoah, p. 168 et p. 172.

31. Hilberg, "La bureaucratie de la solution finale ", p. 222 et p. 230.

32. Ibid. p. 232 et Claude Lanzmann, Shoah, p. 172.

33. Hilberg, "La destruction des Juifs d'Europe », p. 886. 
34. Lorsque Hilberg dans ses conclusions décrit le bureaucrate comme un homme de devoir sans adhésion idéologique fanatique et sans haine particulière contre les Juifs, il n'en rejette pas moins la notion de banalité du mal (sans citer d'ailleurs explicitement Arendt), jugeant que ces bureaucrates de l'Allemagne nazie «travaillaient pour l'histoire et étaient conscients de leur rôle dans cette entreprise ». Cf. Hilberg, "La bureaucratie de la solution finale », p. 233. 